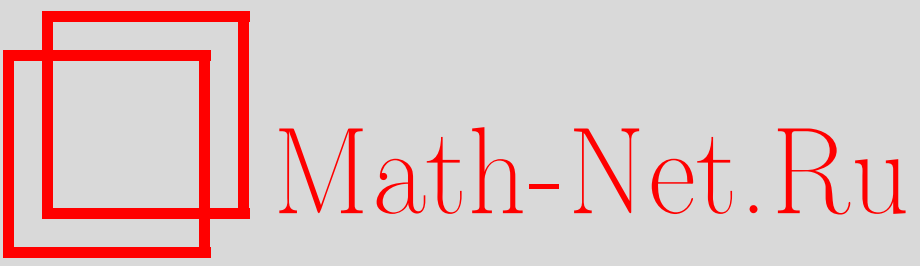

Н. Р. Хуснутдинов, Квазиклассические кротовые норы с гладкой горловиной, TMФ, 2004, том 138, номер 2, 297-318

DOI: https://doi.org/10.4213/tmf20

Использование Общероссийского математического портала Math-Net.Ru подразумевает, что вы прочитали и согласны с пользовательским соглашением

http://www . mathnet.ru/rus/agreement

Параметры загрузки:

IP: 44.207 .124 .84

26 апреля 2023 г., 05:00:29 
ТЕОРЕТИЧЕСКАЯ

И МАТЕМАТИЧЕСКАЯ

ФИЗИКА

Том 138, № 2

февраль, 2004

(C) 2004 г.

\section{КВАЗИКЛАССИЧЕСКИЕ КРОТОВЫЕ НОРЫ С ГЛАДКОЙ ГОРЛОВИНОЙ}

Предложена процедура вычисления коэффициентов ядра теплопроводности, на основе которой получено выражение для любого коэффициента $B_{i}$ ядра теплопроводности спроизвольным профилем горловины. Приведен явный вид этих коэффициентов до $B_{3}$ включительно. Изучены области возможного существования кротовых нор для различных значений отношения длины к радиусу горловины и константы неминимальной связи.

Ключевые слова: энергия нулевых колебаний, дзета-функция, кротовые норы, квантовая теория поля в искривленном пространстве-времени.

\section{1. ВВЕДЕНИЕ}

Интерес к пространству-времени кротовых нор появился достаточно давно. Одна из первых работ была опубликована еще в 1916 году [1]. Последуюшая активность в этом направлении была связана с классической работой Эйнштейна и Розена [2] в контексте пространственно-временной структуры черных дыр и серией работ Уилера [3] для объяснения "всего из ничего". Вновь возникший интерес к задаче кротовых нор связан с работами Морриса, Торна и Юртсевера [4], использовавших эти объекты при научном обсуждении проблемы "машины времени". Они сконструировали и исследовали класс объектов - проходимые кротовые норы. Эти работы повлекли за собой бурную активность в физике кротовых нор [5].

Главная проблема проходимых кротовых нор связана с тем, что они нарушают энергетические условия. Это означает, что материя, создаюшая такие норы, должна обладать экзотическими свойствами. По этой причине кротовую нору нельзя получить как самосогласованное решение уравнений Эйнштейна с источником в виде классической материи, поскольку обычная материя удовлетворяет всем условиям энергодоминантности. Один из возможных способов решения этой проблемы состоит в использовании квантованных полей в рамках полуклассической квантовой гравитации. Дело в том, что вакуумное среднее тензора энергии-импульса квантовых флуктуаций тоже может

* Казанский государственный педагогический университет, Казань, Россия. E-mail: nk@dtp.ksu.ras.ru 
нарушать энергетические условия. Самосогласованные кротовые норы в рамках полуклассической квантовой гравитации изучались в работах [6]. В недавней статье [7] была исследована возможность сушествования решения полуклассических уравнений Эйнштейна, описывающего специальный вид кротовой норы с бесконечно короткой горловиной. Модель представляет собой две одинаковые копии пространства Минковского. Из каждого пространства удалены две сферические полости, границы которых отождествлены. Пространство-время такой модели всюду плоское, за исключением двухмерной сингулярной сферической поверхности. Источником этого пространства является вакуумное среднее квантовых флуктуаций массивного скалярного поля с неминимальной связью. Поскольку пространство-время является фактически везде плоским, стало возможным построение полного набора решений волнового уравнения для массивного скалярного поля и вычисление энергии нулевых колебаний. Для такой модели была вычислена полная энергия квантовых фллукуаций и использованы уравнения Эйнштейна с квантовым источником. Было показано, что энергия флуктуаций, как функция радиуса горловины $a$, может иметь минимум, если константа неминимальной связи $\xi>0.123$. Использование уравнений Эйнштейна в минимуме энергии дает стабильную конфигурацию кротовой норы. Например, в случае конформной связи, $\xi=1 / 6$, радиус $a$ кротовой норы и масса $m$ скалярного поля связаны соотношением $a m \approx 0.16$. Уравнения Эйнштейна дают дополнительную связь параметров, используя которую получаем, что $a \approx 0.0141 l_{\mathrm{Pl}}$ и $m \approx 11.35 m_{\mathrm{Pl}}$. Таким образом, этот тип кротовых нор, если он существует, должен иметь субпланковский размер горловины и должен порождаться квантовыми флуктуациями скалярного поля сверхпланковской массы. Очевидно, что этот результат ограничен выбранной моделью кротовой норы.

Целью данной работы является рассмотрение возможности существования полуклассических кротовых нор с более реальной геометрией горловины и с энергией нулевых колебаний в качестве источника такого пространства. Основная проблема в этом случае носит математический характер. Даже для простейшего профиля горловины становится невозможным получение полного набора решений радиального уравнения, и по этой причине невозможно вычисление в явном виде плотности энергии фллуктуаций. Тем не менее возможно предсказать существование кротовых нор, рассматривая коэффициенты ядра теплопроводности оператора Лапласа [7]. Фактически основным моментом является существование отрицательного минимума энергии нулевых колебаний. Достаточным условием того, что энергия нулевых колебаний имеет отрицательный минимум, является положительность коэффициентов ядра теплопроводности $B_{2}$ и $B_{3}$ [7]. Это приводит к ограничениям на параметры модели. Точнее, если фоновое пространство описывается параметром $\tau$ с размерностью длины и размер области, где пространство "в основном" искривлено, определяется этим параметром, то для малых $(\tau \rightarrow 0)$ и больших $(\tau \rightarrow \infty)$ размеров искривленной области энергия нулевых колебаний ведет себя следующим образом:

$$
E_{\tau \rightarrow 0}^{\mathrm{ren}} \approx-\frac{B_{2} \ln (\tau m)^{2}}{32 \pi^{2}}, \quad E_{\tau \rightarrow \infty}^{\mathrm{ren}} \approx-\frac{B_{3}}{32 \pi^{2} m^{2}}
$$


Условия положительности коэффициентов $B_{2}$ и $B_{3}$ ядра теплопроводности означают, что при малых $\tau$ энергия велика и положительна, а при больших $\tau$ энергия стремится к нулю со стороны отрицательных значений. Очевидно, что в этом случае существует отрицательный минимум энергии. Если оба эти условия выполняются, то можно ожидать, что система будет находиться в минимуме энергии, который характеризуется конкретными значениями параметров кротовой норы и константы неминимальной связи $\xi$.

Следующий шаг состоит в использовании уравнений Эйнштейна с тензором энергии-импульса квантовых флуктуаций в качестве источника. Интегрирование по объему $t-t$ уравнения Эйнштейна дает дополнительное соотношение между параметрами кротовой норы и энергией нулевых колебаний. Используя это соотношение, получаем размер кротовой норы и массу скалярного поля. С самого начала следует ожидать [7], что размер кротовой норы и масса поля будут порядка планковских. По этой причине мы интересуемся только нахождением областей параметров кротовой норы и константы неминимальной связи $\xi$, в которых возможно существование кротовых нор. Следует подчеркнуть, что мы не даем решения полуклассических уравнений Эйнштейна, а лишь приводим доводы в пользу сушествования такого решения.

Для коэффициента $B_{2}$ явные выражения сушествуют для произвольного фонового пространства, но для $B_{3}$ это не так. По этой причине в работе использован метод дзета-регуляризации (см. раздел 3), в рамках которого возможно вычислить коэффициенты ядра теплопроводности и энергию нулевых колебаний. Преследуется также и другая цель - развить подход дзета-регуляризации для ситуаций, где невозможно найти в явном виде полный набор решений радиального уравнения. Такой метод расчета для пространства кротовой норы с произвольным профилем горловины на основе подхода ВКБ изложен в разделе 4. Более того, в рамках такого подхода в разделе 5 найдены формулы для любого коэффициента ядра теплопроводности в случае произвольного профиля горловины.

В статье использованы единишы $\hbar=c=G=1$. Сигнатура пространства-времени, знаки тензора кривизны такие же, как в книге Хокинга и Эллиса [8].

\section{2. ПРОСТРАНСТВО-ВРЕМЯ КРОТОВОЙ НОРЫ}

Элемент длины пространства-времени кротовой норы имеет следующий вид:

$$
d s^{2}=-d t^{2}+d \rho^{2}+r^{2}(\rho)\left(d \theta^{2}+\sin ^{2} \theta d \varphi^{2}\right)
$$

Радиальная переменная $\rho$ изменяется от $-\infty$ до $+\infty$. В данной работе мы ограничимся рассмотрением кротовых нор с симметричной горловиной: $r(-\rho)=r(\rho)$. Радиус горловины $a=r(0)$. Вне горловины кротовой норы пространство-время переходит в плоское пространство-время Минковского, т.е.

$$
\lim _{\rho \rightarrow \pm \infty} \frac{r^{2}(\rho)}{\rho^{2}}=1 .
$$


Ненулевые компоненты тензора Риччи и скалярная кривизна имеют вид

$$
\mathcal{R}_{\rho}^{\rho}=-\frac{2 r^{\prime \prime}}{r}, \quad \mathcal{R}_{\theta}^{\theta}=\mathcal{R}_{\varphi}^{\varphi}=-\frac{-1+r^{\prime 2}+r r^{\prime \prime}}{r^{2}}, \quad \mathcal{R}=-\frac{2\left(-1+r^{\prime 2}+2 r r^{\prime \prime}\right)}{r^{2}}
$$

Источником пространства (1) является материя со следуюшими значениями плотности энергии и давления:

$$
\varepsilon=-\frac{-1+r^{\prime 2}+2 r r^{\prime \prime}}{8 \pi r^{2}}, \quad p_{\rho}=\frac{-1+r^{2}}{8 \pi r^{2}}, \quad p_{\theta}=p_{\varphi}=\frac{r^{\prime \prime}}{8 \pi r}
$$

В данной работе мы получим обшие формулы для коэффициентов ядра теплопроводности в пространстве (1) с произвольной симметричной функцией $r(\rho)$, удовлетворяющей условию (2). В качестве примеров можно привести следующие профили горловины.

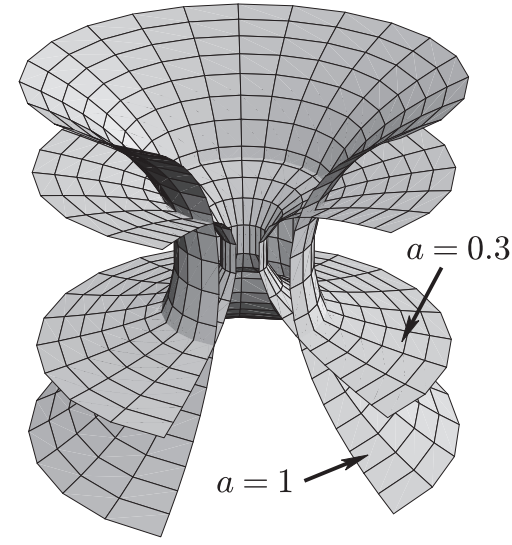

a

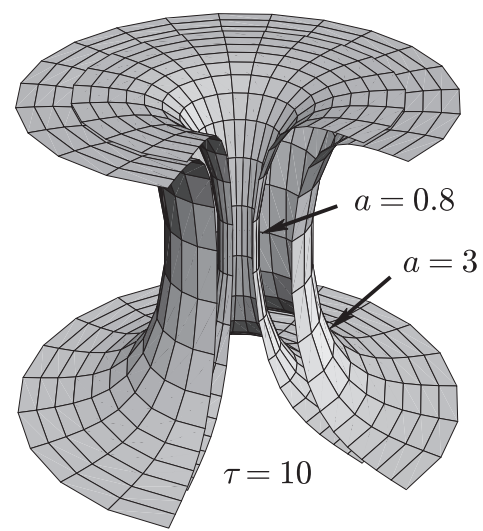

B

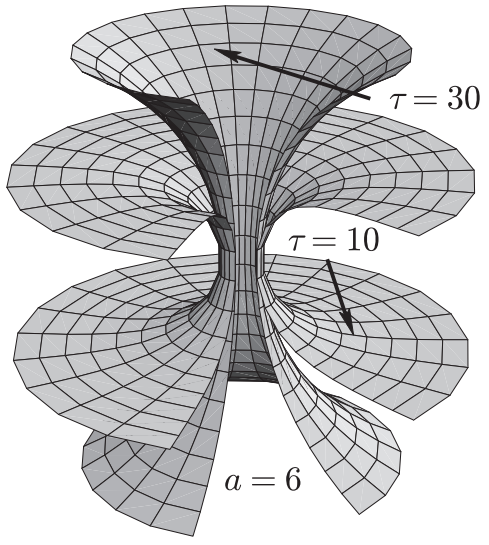

б

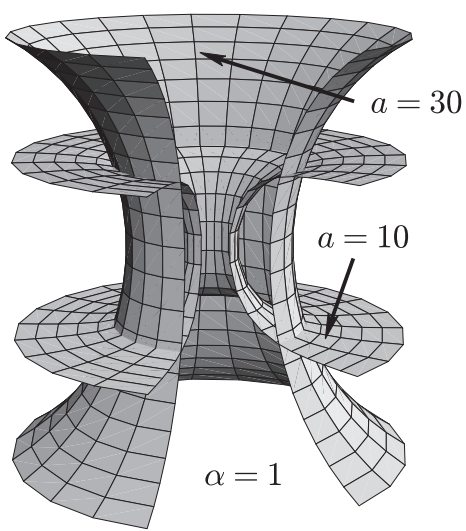

$\Gamma$

Рис. 1 
1. Однопараметрическая модель:

$$
r(\rho)=\sqrt{\rho^{2}+a^{2}} .
$$

Здесь $a$ является радиусом горловины и характеризует кротовую нору в целом. На рис. 1а изображено вложение в трехмерное евклидово пространство сечения пространства (1), (3) плоскостью $t=$ const, $\theta=\pi / 2$ для двух различных радиусов горловины.

2. Двухпараметрическая модель [9]:

$$
r(\rho)=\rho \operatorname{cth} \frac{\rho}{\tau}-\tau+a .
$$

Параметр $a$ является радиусом горловины, поскольку $a=\lim _{\rho \rightarrow 0} r(\rho)$. Параметр $\tau$ описывает длину горловины. Действительно, функция $r(\rho)$ становится линейной функцией $\rho$ начиная с расстояний $\rho>\tau / 2$, и пространство-время переходит в пространство-время Минковского. Поэтому длина горловины $l=\tau$. Используя новые переменные $y=\rho / a, \alpha=\tau / a$, можно переписать функцию $r$ в виде

$$
r(y)=a\left(y \operatorname{cth} \frac{y}{\alpha}-\alpha+1\right) .
$$

Параметр $\alpha$ является отношением длины горловины к ее радиусу. Таким образом, эта модель позволяет рассмотреть кротовые норы различной формы (параметр $\alpha$ ) и различного размера (параметр $a$ ).

На рис. $16-1$ г изображены сечения $t=$ const, $\theta=\pi / 2$ пространства-времени кротовой норы для различных значений параметров $a$ и $\tau$. Две кротовые норы с одинаковым радиусом горловины, но с различной ее длиной изображены на рис. $1 б$, а на рис. 1в показаны две кротовые норы с одинаковой длиной горловины, но с различными ее радиусами. На рис. 1г изображены две кротовые норы с одинаковым отношением радиуса к длине горловины, но с различными значениями ее длины.

В данной работе мы рассмотрим другую двухпараметрическую модель кротовой норы со следуюшим профилем горловины:

$$
r(\rho)=\rho \operatorname{th} \frac{\rho}{\tau}+a .
$$

Параметры $a$ и $\tau$ имеют такой же смысл, как и в модели (4).

\section{3. ЭНЕРГИЯ НУЛЕВЫХ КОЛЕБАНИЙ. МЕТОД ДЗЕТА-ФУНКЦИИ}

Для вычисления энергии нулевых колебаний массивного скалярного поля в пространстве кротовой норы (1) используется метод дзета-регуляризации, предложенный первоначально в работах [10], [11] и развитый в [12]. Приведем основные формулы из этих работ. В рамках подхода энергия нулевых колебаний

$$
E(s)=\frac{1}{2} \mu^{2 s} \sum_{j} \sum_{(n)}\left(\lambda_{(n), j}^{2}+m^{2}\right)^{1 / 2-s}=\frac{1}{2} \mu^{2 s} \zeta_{\mathcal{L}}\left(s-\frac{1}{2}\right)
$$


массивного скалярного поля $\Phi$ выражается через дзета-функцию

$$
\zeta_{\mathcal{L}}\left(s-\frac{1}{2}\right)=\sum_{j} \sum_{(n)}\left(\lambda_{(n), j}^{2}+m^{2}\right)^{1 / 2-s}
$$

трехмерного оператора Лапласа $\mathcal{L}=-\Delta+m^{2}+\xi \mathcal{R}$. Здесь $\Delta=g^{k l} \nabla_{k} \nabla_{l}-$ трехмерный оператор Бельтрами. Собственные значения $\lambda_{(n), j}+m^{2}$ оператора $\mathcal{L}$ находятся из граничных условий

$$
\Psi_{(n)}(\lambda, R)=0,
$$

где $R$ обозначает параметр, характеризуюший границу. Решения $\lambda=\lambda_{(n), j}$ этого уравнения зависят от чисел $(n)$ и имеют дополнительный индекс $j=1,2, \ldots$, который нумерует решения граничного условия. Теперь в соответствии с [12] конвертируем ряд по $j$ в выражении для дзета-функции (7) в интеграл,

$$
\zeta_{\mathcal{L}}\left(s-\frac{1}{2}\right)=-\sum_{(n)} \frac{\cos \pi s}{\pi} \int_{m}^{\infty} d k\left(k^{2}-m^{2}\right)^{1 / 2-s} \frac{\partial}{\partial k} \ln \Psi_{(n)}(i k, R) .
$$

Заметим, что функция $\Psi_{(n)}$ вычисляется на мнимой оси.

В интересуюшем нас пределе $s \rightarrow 0$ выражение (9) расходится. Для перенормировки вычитаем из $E(s)$ все члены $E^{\operatorname{div}}(s)$, которые остаются в пределе $m \rightarrow \infty$,

$$
E^{\operatorname{div}}(s)=\lim _{m \rightarrow \infty} E(s)
$$

и определяем перенормированную энергию следуюшим образом:

$$
E^{\mathrm{ren}}=\lim _{s \rightarrow 0}\left(E(s)-E^{\operatorname{div}}(s)\right)
$$

Поскольку полюсная структура дзета-функции не зависит от величины параметров, то очевидно, что в пределе $m \rightarrow \infty$ расходяшаяся часть имеет вид разложения Девитта-Швингера

$$
\begin{aligned}
E^{\operatorname{div}}(s)= & \frac{1}{2}\left(\frac{\mu}{m}\right)^{2 s} \frac{1}{(4 \pi)^{3 / 2} \Gamma\left(s-\frac{1}{2}\right)}\left\{B_{0} m^{4} \Gamma(s-2)+B_{1 / 2} m^{3} \Gamma\left(s-\frac{3}{2}\right)+\right. \\
& \left.+B_{1} m^{2} \Gamma(s-1)+B_{3 / 2} m \Gamma\left(s-\frac{1}{2}\right)+B_{2} \Gamma(s)\right\}
\end{aligned}
$$

где $B_{\alpha}$ - коэффициенты ядра теплопроводности. Для того чтобы выделить расходящуюся часть энергии, используем следующую процедуру [12]. Вычитаем и добавляем к подынтегральному выражению равномерное разложение $\ln \Psi$, в котором учтены все слагаемые до $m^{0}$. Обозначим это разложение через $\left(\ln \Psi_{(n)}\right)$ as . В соответствии с этим представим энергию в виде суммы двух слагаемых,

$$
E(s)=E_{\mathrm{fin}}(s)+E_{\mathrm{as}}(s),
$$


где $E_{\mathrm{fin}}(s)$ - конечная часть (в пределе $s \rightarrow 0$ ),

$$
\begin{aligned}
E_{\mathrm{fin}}(s)= & -\frac{1}{2} \mu^{2 s} \sum_{(n)} \frac{\cos \pi s}{\pi} \int_{m}^{\infty} d k\left(k^{2}-m^{2}\right)^{1 / 2-s} \times \\
& \times \frac{\partial}{\partial k}\left(\ln \Psi_{(n)}(i k, R)-\left(\ln \Psi_{(n)}(i k, R)\right)^{\text {as }}\right),
\end{aligned}
$$

a $E_{\mathrm{as}}(s)$ - остаток, который получается из равномерного разложения,

$$
E_{\text {as }}(s)=-\frac{1}{2} \mu^{2 s} \sum_{(n)} \frac{\cos \pi s}{\pi} \int_{m}^{\infty} d k\left(k^{2}-m^{2}\right)^{1 / 2-s} \frac{\partial}{\partial k}\left(\ln \Psi_{(n)}(i k, R)\right)^{\text {as }} .
$$

Последнее выражение содержит все члены, которые остаются в пределе $m \rightarrow \infty$.

Используя полученные соотношения в выражении (10), получаем

$$
\begin{aligned}
& E^{\mathrm{ren}}=E_{\mathrm{fin}}+E_{\mathrm{as}}^{\mathrm{fin}}, \\
& E_{\mathrm{fin}}=E_{\mathrm{fin}}(0)=-\frac{1}{2 \pi} \sum_{(n)} \int_{m}^{\infty} d k \sqrt{k^{2}-m^{2}} \frac{\partial}{\partial k}\left(\ln \Psi_{(n)}(i k, R)-\left(\ln \Psi_{(n)}(i k, R)\right)^{\mathrm{as}}\right), \\
& E_{\mathrm{as}}^{\mathrm{fin}}=\lim _{s \rightarrow 0}\left(E_{\mathrm{as}}(s)-E^{\mathrm{div}}(s)\right) .
\end{aligned}
$$

Конечная часть $E_{\text {fin }}$ вычисляется численно. Вторая часть находится следующим образом. Используя равномерное разложение $\left(\ln \Psi_{(n)}\right)$ as , вычисляем в явном виде функцию $E_{\text {as }}(s)$ и затем берем предел $m \rightarrow \infty$ в полученном выражении (полюсная структура не изменяется). Все слагаемые, которые остаются в этом пределе, представляют собой разложение Девитта-Швингера (11), которое вычитается в уравнении (13в). Вычисление коэффициентов в рамках этой процедуры показывает, что такой метод подходит не только для гладких многообразий, но и для пространств, содержащих сингулярные поверхности коразмерности один [7] и два [13], общие формулы для которых получены в работах [14] и [15].

Используемый подход позволяет качественно проанализировать поведение энергии нулевых колебаний для больших и малых размеров кротовых нор [7]. Пусть параметр $а$ характеризует размер кротовой норы. Тогда $E^{\mathrm{ren}} / m$ является безразмерной функцией, зависящей от параметра та и некоторых дополнительных безразмерных параметров, которые характеризуют форму кротовой норы. Например, в первой модели (3) есть только один параметр $a$, который является радиусом кротовой норы и в тоже время характеризует ее размер. Таким образом, в этой модели $E^{\mathrm{ren}} / m$ зависит только от $m a$. Во второй (4) и третьей (5) моделях кроме та имеется дополнительный параметр $\alpha=\tau / a$. Поэтому зависимость энергии нулевых колебаний $E^{\mathrm{ren}} / m$ от массы такая же, как и от параметра $a$. Поскольку для перенормировки мы вычли все члены асимптотического разложения до $B_{2}$, то асимптотика $m a \rightarrow \infty$ энергии принимает вид

$$
\frac{E^{\text {ren }}}{m} \approx-\frac{B_{3}}{32 \pi^{2} m^{3}}=-\frac{b_{3}}{32 \pi^{2}(m a)^{3}} .
$$


В случае $m a \rightarrow 0$ поведение энергии описывается коэффициентом $B_{2}$,

$$
\frac{E^{\mathrm{ren}}}{m} \approx-\frac{\ln (m a)^{2}}{32 \pi^{2} m} B_{2}=-\frac{\ln (m a)^{2}}{32 \pi^{2}(m a)} b_{2} .
$$

Здесь $b_{3}$ и $b_{2}$ являются безразмерными коэффициентами ядра теплопроводности, которые могут зависеть от дополнительных параметров, характеризуюших форму кротовой норы. Из этих выражений получаем следующее достаточное условие того, что энергия нулевых колебаний имеет минимум: $B_{2}$ и $B_{3}$ должны быть положительными. Дополнительное условие может быть получено из уравнений Эйнштейна.

Функция $\Psi_{(n)}(i k, R)$ обычно имеет сложный вид или вообще не может быть выражена через известные функции. Поэтому интегрирование в (9) невозможно провести в явном виде. Для нахождения коэффициентов ядра теплопроводности можно использовать в $(9)$ равномерное разложение функции $\Psi_{(n)}(i k, R)$ по обратным степеням индекса $(n)$ с последуюшим взятием предела $m \rightarrow \infty$. Такая процедура неоднократно применялась в различных ситуациях как для гладких многообразий [7], [12], [13], [16], так и для многообразий, содержаших сингулярные поверхности коразмерности один и два $[14],[15]$. В случае пространства кротовой норы $(1)$ функция $\Psi_{(n)}(i k, R)$ не выражается через известные функции, и по этой причине ниже будет развита процедура нахождения коэффициентов ядра теплопроводности, не используюшая в явном виде функцию $\Psi_{(n)}(i k, R)$.

\section{4. МАССИВНОЕ СКАЛЯРНОЕ ПОЛЕ В ПРОСТРАНСТВЕ КРОТОВОЙ НОРЫ В РАМКАХ ВКБ-ПОДХОДА}

Рассмотрим спектральную задачу для оператора Лапласа $\mathcal{L}$,

$$
(-\Delta+\xi \mathcal{R}) \Phi=\lambda \Phi .
$$

Используя сферическую симметрию задачи, представим решение уравнения в виде

$$
\Phi=Y_{l}^{m}(\theta, \varphi) \phi
$$

где $Y_{l}^{m}(\theta, \varphi)$ являются сферическими гармониками, $l=0,1,2, \ldots, m \in[-l, l]$. Радиальная часть волновой функции удовлетворяет уравнению

$$
\left(\partial_{\rho}^{2}+\frac{2 r^{\prime}}{r} \partial_{\rho}-\frac{l(l+1)}{r^{2}}-\xi \mathcal{R}\right) \phi=-\lambda^{2} \phi
$$

Решение этого уравнения не выражается через известные функции даже для простейших видов профиля горловины (3), (4). По этой причине получим равномерное разложение требуемых решений, не используя их явный вид.

Для нахождения спектра необходимо наложить какие-либо граничные условия, которые и определят функцию $\Psi_{(n)}(i k, R)$. Мы используем простейшие условия Дирихле на 
двух сферах радиуса $R: \rho= \pm R$. Поскольку в рамках используемого подхода нам необходимо получить решение радиального уравнения на мнимой оси (для мнимой энергии), то в уравнении (16) заменяем $\lambda \rightarrow i \nu k$ и для упрошения формул переходим к новой переменной $\rho k \rightarrow x$. Таким образом, получаем следуюшее уравнение $(\nu=l+1 / 2)$ :

$$
\ddot{\phi}+2 \frac{\dot{r_{k}}}{r_{k}} \dot{\phi}-\nu^{2}\left(1+\frac{1}{r_{k}^{2}}\right) \phi+\left(\frac{1}{4 r_{k}^{2}}-\xi \mathcal{R}_{k}\right) \phi=0,
$$

где точка обозначает производную по $x, r_{k}^{2}=r^{2} k^{2}, \mathcal{R}_{k}=\mathcal{R} / k^{2}$.

Общим решением радиального уравнения (17) является суперпозиция двух линейно-независимых решений

$$
\bar{\Psi}(i \nu k, x)=C_{1} \phi_{1}(x)+C_{2} \phi_{2}(x) .
$$

Первая функция $\phi_{1}$ стремится к бесконечности в удаленной от горловины области $(\rho \rightarrow \infty)$, а вторая $\phi_{2}$ стремится к нулю. Рассмотрим поведение функций только в одной половине пространства при $\rho>0$. Поведение решений во второй части с отрицательным $\rho$ получается продолжением решений для положительных $\rho$. Потребуем выполнения граничных условий Дирихле на сферах $\rho= \pm R$,

$$
\begin{aligned}
\bar{\Psi}(i \nu k, R) & =C_{1} \phi_{1}(R)+C_{2} \phi_{2}(R)=0, \\
\bar{\Psi}(i \nu k,-R) & =C_{1} \phi_{1}(-R)+C_{2} \phi_{2}(-R)=0 .
\end{aligned}
$$

Нетривиальное решение этой системы существует тогда и только тогда, когда главньй определитель системы равен нулю, т.е.

$$
\Psi_{l}(i \nu k, R)=\phi_{1}(R) \phi_{2}(-R)-\phi_{1}(-R) \phi_{2}(R)=0 .
$$

Вклад второго слагаемого в этом уравнении экспоненциально мал по сравнению с первым в пределе $R \rightarrow \infty$. Это будет ясно после получения равномерного разложения решений $\phi_{1}$ и $\phi_{2}$. Для этого представим решение $\phi$ в экспоненциальной форме

$$
\phi(x)=\frac{1}{\sqrt{2 a \nu}} e^{S(x)},
$$

где $a=r(0)$, и подставим в радиальное уравнение (17). Получаем нелинейное уравнение

$$
\ddot{S}+\dot{S}^{2}+2 \frac{\dot{r_{k}}}{r_{k}} \dot{S}-\nu^{2}\left(1+\frac{1}{r_{k}^{2}}\right)+\left(\frac{1}{4 r_{k}^{2}}-\xi \mathcal{R}_{k}\right)=0
$$

Представляя решение в форме ВКБ

$$
S=\sum_{n=-1}^{\infty} \nu^{-n} S_{n}
$$

5 Теоретическая и математическая физика, т. 138, № 2, 2004 г. 
получаем следующую цепочку уравнений:

$$
\begin{aligned}
\dot{S}_{-1} & = \pm \sqrt{1+\frac{1}{r_{k}^{2}}}, \\
\dot{S}_{0} & =-\frac{1}{2} \frac{\ddot{S}_{-1}}{\dot{S}_{-1}}-\frac{\dot{r}_{k}}{r_{k}} \\
\dot{S}_{1} & =-\frac{1}{2 \dot{S}_{-1}}\left[\ddot{S}_{0}+\dot{S}_{0}^{2}+2 \frac{\dot{r}_{k}}{r_{k}} \dot{S}_{0}+\frac{1}{4 r_{k}^{2}}-\xi \mathcal{R}_{k}\right], \\
\dot{S}_{n+1} & =-\frac{1}{2 \dot{S}_{-1}}\left[\ddot{S}_{n}+\sum_{k=0}^{n} \dot{S}_{k} \dot{S}_{n-k}+2 \frac{\dot{r}_{k}}{r_{k}} \dot{S}_{n}\right], \quad n=1,2, \ldots
\end{aligned}
$$

Существуют два решения этой цепочки, соответствующие знаку первого уравнения. Знак плюс приводит к растушему (для положительных $\rho$ ) решению, которое будем помечать знаком “+", и знак минус приводит к решению, которое стремится к нулю на бесконечности. Его будем помечать знаком “-”. Таким образом,

$$
\phi_{1}(R) \phi_{2}(-R)=\frac{1}{2 a \nu} e^{S^{+}(R)+S^{-}(-R)}
$$

Для того чтобы получить разложение для суммы $S^{+}(R)+S^{-}(-R)$, необходимо использовать следующие свойства функций $S^{ \pm}(x)$ :

$$
\begin{aligned}
\dot{S}_{2 n-1}^{-}(x) & =-\dot{S}_{2 n-1}^{+}(x), \\
\dot{S}_{2 n}^{-}(x) & =\dot{S}_{2 n}^{+}(x), \\
\dot{S}_{2 n-1}^{ \pm}(x) & =\dot{S}_{2 n-1}^{ \pm}(-x), \\
\dot{S}_{2 n}^{ \pm}(x) & =-\dot{S}_{2 n}^{ \pm}(-x),
\end{aligned}
$$

где $n=0,1, \ldots$. Первые два соотношения являются следствием структуры цепочки уравнений, а два последние - следствием симметрии метрической функции $r_{k}(x)=$ $r_{k}(-x)$. Используя эти свойства, получаем

$$
\begin{aligned}
S^{+}(x)+S^{-}(-x)= & \sum_{n=0}^{\infty} \nu^{1-2 n}\left[C_{2 n-1}^{+}+C_{2 n-1}^{-}+\int_{-x}^{+x} \dot{S}_{2 n-1}^{+} d x\right]+ \\
& +\sum_{n=0}^{\infty} \nu^{-2 n}\left[C_{2 n}^{+}+C_{2 n}^{-}+2 \int_{x_{0}}^{+x} \dot{S}_{2 n}^{+} d x\right] \\
S^{+}(x)+S^{-}(x)= & \sum_{n=0}^{\infty} \nu^{1-2 n}\left[C_{2 n-1}^{+}+C_{2 n-1}^{-}\right]+ \\
& +\sum_{n=0}^{\infty} \nu^{-2 n}\left[C_{2 n}^{+}+C_{2 n}^{-}+2 \int_{x_{0}}^{+x} \dot{S}_{2 n}^{+} d x\right]
\end{aligned}
$$


Здесь $C_{n}^{ \pm}$являются постоянными интегрирования системы (21). Вычитая одно уравнение из другого, исключаем из рассмотрения константы,

$$
S^{+}(x)+S^{-}(-x)=S^{+}(x)+S^{-}(x)+\sum_{n=0}^{\infty} \nu^{1-2 n} \int_{-x}^{+x} \dot{S}_{2 n-1}^{+} d x .
$$

Для того чтобы найти комбинацию $S^{+}(x)+S^{-}(x)$, используем условие линейной независимости решений. Это эквивалентно тому, что вронскиан решений отличен от нуля,

$$
W\left(\phi_{1}(x), \phi_{2}(x)\right)=\frac{k}{r_{k}^{2}} .
$$

Происхождение этого соотношения следуюшее. Предположим, что мы ишем функцию Грина скалярного уравнения Клейна-Гордона-Фока

$$
\left(g^{\mu \nu} \nabla_{\mu} \nabla_{\nu}-m^{2}-\xi \mathcal{R}\right) G\left(x, x^{\prime}\right)=\frac{\delta^{4}\left(x, x^{\prime}\right)}{\sqrt{-g(x)}}
$$

в пространстве с метрикой (1). Легко отделить временну́ю и угловую зависимости функции Грина

$$
G\left(x, x^{\prime}\right)=\int_{-\infty}^{+\infty} \frac{d \omega}{2 \pi} \sum_{l=0}^{\infty} \sum_{m=-l}^{l} Y_{l}^{m}(\theta, \varphi) Y_{l}^{-m}\left(\theta^{\prime}, \varphi^{\prime}\right) e^{-i \omega\left(t-t^{\prime}\right)} \phi\left(\rho, \rho^{\prime}\right) .
$$

Радиальная часть функции Грина удовлетворяет уравнению $\left(\lambda^{2}=\omega^{2}-m^{2}\right)$

$$
\left\{\partial_{\rho}^{2}+\frac{2 r^{\prime}}{r} \partial_{\rho}+\lambda^{2}-\frac{l(l+1)}{r^{2}}-\xi \mathcal{R}\right\} \phi\left(\rho, \rho^{\prime}\right)=\frac{\delta\left(\rho-\rho^{\prime}\right)}{r^{2}},
$$

или в безразмерных переменных $(\lambda \rightarrow i \nu k, k \rho \rightarrow x)-$

$$
\left\{\partial_{x}^{2}+2 \frac{\dot{r_{k}}}{r_{k}} \partial_{x}-\nu^{2}\left(1+\frac{1}{r_{k}^{2}}\right)+\left(\frac{1}{4 r_{k}^{2}}-\xi \mathcal{R}_{k}\right)\right\} \phi\left(x, x^{\prime}\right)=\frac{k \delta\left(x-x^{\prime}\right)}{r_{k}^{2}} .
$$

Представляем радиальную часть функции Грина в стандартной форме

$$
\phi\left(x, x^{\prime}\right)=\theta\left(x^{\prime}-x\right) \phi_{1}(x) \phi_{2}\left(x^{\prime}\right)+\theta\left(x-x^{\prime}\right) \phi_{2}(x) \phi_{1}\left(x^{\prime}\right),
$$

где $\phi_{1}$ и $\phi_{2}$ являются линейно-независимыми решениями однородного уравнения, $\phi_{1}$ стремится к бесконечности, а $\phi_{2}$ стремится к нулю для $\rho \rightarrow \infty$. Условие на вронскиан (24) возникает при подстановке этой формы радиальной функции Грина в радиальное уравнение. Для решений в экспоненциальной форме (20) это условие дает $\left(a_{k}=a k=\right.$ $\left.r_{k}(0)\right)$

$$
e^{S^{+}(x)+S^{-}(x)}=\frac{2 \nu a_{k}}{r_{k}^{2}} \frac{1}{\dot{S}^{+}(x)-\dot{S}^{-}(x)} .
$$


ВКБ-разложение знаменателя в правой части имеет вид

$$
\dot{S}^{+}(x)-\dot{S}^{-}(x)=2 \sum_{n=0}^{\infty} \nu^{1-2 n} \dot{S}_{2 n-1}^{+}
$$

Используя полученные выражения, получаем следующую формулу:

$$
\begin{aligned}
S^{+}(x)+S^{-}(-x)= & \ln a_{k}-\frac{1}{2} \ln \left(\dot{S}_{-1}^{2} r_{k}^{4}\right)-\ln \left(1+\sum_{n=1}^{\infty} \nu^{-2 n} \frac{\dot{S}_{2 n-1}^{+}}{\dot{S}_{-1}^{+}}\right)+ \\
& +\sum_{n=0}^{\infty} \nu^{1-2 n} \int_{-x}^{+x} \dot{S}_{2 n-1}^{+} d x
\end{aligned}
$$

Отметим основные особенности этого выражения:

а) правая часть (26) выражается через производные функций $S_{n}^{+}$, т.е. нет нужды находить постоянные интегрирования в цепочке (21);

б) нечетные и четные степени $\nu$ разделились, что ведет к разделению вкладов в коэффициенты ядра теплопроводности с целым и полуцелым индексами;

в) правая часть (26) выражается только через функции $S_{n}$ с нечетными индексами.

Из соотношения (26) видно, что вклад от второго слагаемого в выражении (19) экспоненшиально мал по сравнению с первым. Действительно, главный ВКБ-вкладв уравнении (26) приводит к следуюшим условиям:

$$
\begin{aligned}
& \phi_{1}(R) \phi_{2}(-R) \approx \frac{k}{2 \nu} \frac{1}{\dot{S}_{-1}^{+} r_{k}^{2}} \exp \left(\nu \int_{-k R}^{+k R} \dot{S}_{-1}^{+} d x\right), \\
& \phi_{1}(-R) \phi_{2}(R) \approx \frac{k}{2 \nu} \frac{1}{\dot{S}_{-1}^{+} r_{k}^{2}} \exp \left(-\nu \int_{-k R}^{+k R} \dot{S}_{-1}^{+} d x\right) .
\end{aligned}
$$

Поскольку функция $\dot{S}_{-1}^{+}$положительна для любого $R$, то второе слагаемое в (19) дает экспоненциально малый вклад (для $R \rightarrow \infty$ ) по сравнению с первым. В дальнейшем мы будем им пренебрегать.

\section{5. КОЭФФИЦИЕНТЫ ЯДРА ТЕПЛОПРОВОДНОСТИ}

Формула (26) позволяет вычислить коэффициенты ядра теплопроводности для произвольного индекса. Используя экспоненциальную форму решений, получаем следующее выражение для дзета-функции:

$$
\zeta\left(s-\frac{1}{2}\right)=-m^{2 s} \frac{2 \cos \pi s}{\pi} \sum_{l=0}^{\infty} \nu^{2-2 s} \int_{\frac{m}{\nu}}^{\infty} d k\left(k^{2}-\frac{m^{2}}{\nu^{2}}\right)^{1 / 2-s} \frac{\partial}{\partial k}\left[S^{+}(R)+S^{-}(-R)\right] .
$$

Как будет видно далее, нечетные степени $\nu$ дают вклад в коэффициенты ядра теплопроводности с целыми индексами, а четные степени $\nu$ - в коэффициенты с полуцелыми индексами. По этой причине будем рассматривать отдельно четные и нечетные степени 
разложения, а соответствуюшие части дзета-функции будем помечать индексами еven и odd. Хорошо известное асимптотическое разложение дзета-функции в трех измерениях имеет следуюший вид:

$$
\zeta_{\text {as }}\left(s-\frac{1}{2}\right)=\frac{(4 \pi)^{-3 / 2}}{\Gamma\left(s-\frac{1}{2}\right)} \sum_{l=0}^{\infty}\left[m^{4-2 l} B_{l} \Gamma(s+l-2)+m^{3-2 l} B_{l+1 / 2} \Gamma\left(s+l-\frac{3}{2}\right)\right] .
$$

Для упрошения формул определим плотности $\bar{B}_{l}$ коэффишиентов ядра теплопроводности соотношением

$$
B_{l}=\int_{-R}^{+R} d \rho \bar{B}_{l}(\rho)
$$

и получим выражения для них.

Рассмотрим сначала часть уравнения (26) с нечетными степенями $\nu$. Меняя переменную интегрирования $x=k \rho$ и вычисляя производную по $k$, получаем следующий вклад в дзета-функцию:

$$
\begin{aligned}
\zeta_{\mathrm{as}}^{\text {odd }}\left(s-\frac{1}{2}\right)= & -m^{2 s} \frac{2 \cos \pi s}{\pi} \sum_{l=0}^{\infty} \nu^{2-2 s} \times \\
& \times \int_{\frac{m}{\nu}}^{\infty} d k k\left(k^{2}-\frac{m^{2}}{\nu^{2}}\right)^{1 / 2-s} \sum_{p=0}^{\infty} \nu^{1-2 p} \int_{-R}^{+R} s_{2 p-1}(k, \rho) d \rho .
\end{aligned}
$$

Функции $s_{2 p-1}$ имеют следуюшую обшую структуру:

$$
s_{2 p-1}=\sum_{n=0}^{2 p} \alpha_{2 p-1, n} z^{-p-n-\frac{1}{2}}
$$

где коэффициенты $\alpha_{2 p-1, n}$ зависят от $r(\rho)$, а $z=1+k^{2} r^{2}(\rho)$. Интегрируя по $k$, получаем следуюшее выражение для нечетной части дзета-функции:

$$
\zeta_{\mathrm{as}}^{\mathrm{odd}}\left(s-\frac{1}{2}\right)=\frac{m^{2 s}}{\Gamma\left(s-\frac{1}{2}\right)} \int_{-R}^{+R} d \rho \sum_{l=0}^{\infty} \sum_{p=0}^{\infty} \sum_{n=0}^{2 p} \frac{\Gamma(s+p+n-1)}{\Gamma\left(p+n+\frac{1}{2}\right)} \frac{\alpha_{2 p-1, n} r^{2 s-3} \nu^{2 n+1}}{\left(\nu^{2}+m^{2} r^{2}\right)^{s+p+n-1}}
$$

Далее используем бином Ньютона

$$
\sum_{l=0}^{\infty} \frac{\nu^{2 n+1}}{\left(\nu^{2}+m^{2} r^{2}\right)^{s+p+n-1}}=\frac{1}{2} \sum_{q=0}^{n}\left(-m^{2} r^{2}\right)^{n-q} \frac{n !}{q !(n-q) !} \frac{\mathcal{Z}(s+p+n-1-q)}{\Gamma(s+p+n-1-q)}
$$

где

$$
\mathcal{Z}(s)=2 \Gamma(s) \sum_{l=0}^{\infty} \frac{\nu}{\left(\nu^{2}+m^{2} r^{2}\right)^{s}}
$$


Для того чтобы найти коэффициенты ядра теплопроводности, необходимо получить для дзета-функции асимптотический ряд по степеням массы. Асимптотическое разложение функции $\mathcal{Z}(s)$ было получено в работе [16] и имеет следующий вид:

$$
\begin{gathered}
\mathcal{Z}(s)=(m r)^{-2 s} \sum_{l=-1}^{\infty} A_{l}(s)(m r)^{-2 l}, \\
A_{-1}(s)=\Gamma(s-1), \\
A_{l}(s)=2 \frac{(-1)^{l}}{l !} \Gamma(l+s) \zeta_{\mathrm{H}}\left(-1-2 l, \frac{1}{2}\right),
\end{gathered}
$$

где $\zeta_{\mathrm{H}}(a, b)$ является дзета-функцией Гурвица.

Используя формулы (30) и (31), получаем следуюший асимптотический ряд для нечетной части дзета-функции:

$$
\begin{aligned}
\zeta_{\text {as }}^{\text {odd }}\left(s-\frac{1}{2}\right)= & \frac{1}{2 \Gamma\left(s-\frac{1}{2}\right)} \int_{-R}^{+R} d \rho \sum_{l=0}^{\infty} \sum_{p=0}^{l} \sum_{n=0}^{2 p} \sum_{q=0}^{n} \alpha_{2 p-1, n} m^{4-2 l} r^{-2 l+1} \times \\
& \times \frac{\Gamma(p+n-1+s)}{\Gamma\left(p+n+\frac{1}{2}\right)} \frac{n !}{q !(n-q) !}(-1)^{n-q} \frac{A_{l-p-1}(s+p+n-1-q)}{\Gamma(s+p+n-1-q)} .
\end{aligned}
$$

В итоге мы нашли ряд по четным степеням массы, который приводит к вкладу в коэффициенты ядра теплопроводности с целыми индексами. Сравнивая это выражение с обшей формулой (28), получаем формулу для произвольного коэффициента ядра теплопроводности с целым индексом,

$$
\begin{aligned}
\bar{B}_{l}(\rho)= & \frac{4 \pi^{3 / 2} r^{-2 l+1}}{\Gamma(s+l-2)} \sum_{p=0}^{l} \sum_{n=0}^{2 p} \sum_{q=0}^{n} \alpha_{2 p-1, n} \frac{n !(-1)^{n-q}}{q !(n-q) !} \times \\
& \times \frac{\Gamma(p+n-1+s)}{\Gamma\left(p+n+\frac{1}{2}\right)} \frac{A_{l-p-1}(s+p+n-1-q)}{\Gamma(s+p+n-1-q)} .
\end{aligned}
$$

Для того чтобы получить коэффициент ядра теплопроводности с индексом $l$, необходимо учесть разложение до $\nu^{1-2 l}$.

Перейдем теперь к вычислению коэффициентов ядра теплопроводности с полуцелым индексом. Для этого необходимо учесть оставшуюся часть в уравнении $(26)$ с четными степенями $\nu$. Обшая структура четной части имеет вид

$$
\left(S^{+}(x)+S^{-}(-x)\right)^{\text {even }}=\ln a_{k}-\frac{1}{2} \ln \left(\dot{S}_{-1}^{2} r_{k}^{4}\right)-\ln \left(1+\sum_{n=1}^{\infty} \nu^{-2 n} \frac{\dot{S}_{2 n-1}^{+}}{\dot{S}_{-1}^{+}}\right)=\sum_{p=0}^{\infty} \nu^{-2 p} E_{2 p},
$$

где функции $E_{2 p}$ можно легко получить разложением логарифма. Подставляем это разложение в выражение для дзета-функции и берем производную по $k$,

$$
\zeta_{\text {as }}^{\text {even }}\left(s-\frac{1}{2}\right)=-m^{2 s} \frac{2 \cos \pi s}{\pi} \sum_{l=0}^{\infty} \nu^{2-2 s} \int_{\frac{m}{\nu}}^{\infty} d k k\left(k^{2}-\frac{m^{2}}{\nu^{2}}\right)^{1 / 2-s} \sum_{p=0}^{\infty} \nu^{-2 p} s_{2 p} .
$$


Функции $s_{2 p}$ записываются в виде суммы,

$$
s_{2 p}=\sum_{n=0}^{2 p} \alpha_{2 p, n} z^{-p-n-1}
$$

где $z=1+k^{2} r^{2}(R)$. Коэффициенты $\alpha_{2 p, n}$ зависят от параметров $R$ и $a$ и не зависят от переменной интегрирования $k$. Действуя точно так же, как и в случае вычисления коэффициентов ядра теплопроводности с целым индексом, получаем следуюшее асимптотическое разложение для четной части дзета-функции:

$$
\begin{aligned}
\zeta_{\text {as }}^{\text {even }}\left(s-\frac{1}{2}\right)= & \frac{1}{2 \Gamma\left(s-\frac{1}{2}\right)} \sum_{l=0}^{\infty} \sum_{p=0}^{l} \sum_{n=0}^{2 p} \sum_{q=0}^{n} \alpha_{2 p, n} m^{3-2 l} r^{-2 l} \frac{\Gamma\left(p+n-\frac{1}{2}+s\right)}{\Gamma(p+n+1)} \times \\
& \times \frac{n !}{q !(n-q) !}(-1)^{n-q} \frac{A_{l-p-1}\left(s+p+n-\frac{1}{2}-q\right)}{\Gamma\left(s+p+n-\frac{1}{2}-q\right)} .
\end{aligned}
$$

Четная часть дзета-функции является рядом по нечетным степеням массы и дает вклад в коэффициенты ядра теплопроводности с полуцелым индексом. Сравнивая это выражение с общей асимптотической формулой для дзета-фрункции, получаем следующее выражение для коэффициентов ядра теплопроводности с полуцелым индексом:

$$
\begin{aligned}
B_{l+\frac{1}{2}}= & \frac{4 \pi^{3 / 2} r^{-2 l}}{\Gamma\left(s+l-\frac{3}{2}\right)} \sum_{p=0}^{l} \sum_{n=0}^{2 p} \sum_{q=0}^{n} \alpha_{2 p, n} \frac{n !(-1)^{n-q}}{q !(n-q) !} \times \\
& \times \frac{\Gamma\left(p+n-\frac{1}{2}+s\right)}{\Gamma(p+n+1)} \frac{A_{l-p-1}\left(s+p+n-\frac{1}{2}-q\right)}{\Gamma\left(s+p+n-\frac{1}{2}-q\right)} .
\end{aligned}
$$

Необходимо подчеркнуть, что правые части формул (32) и (34) фактически не зависят от $s$, что и подтверждается непосредственными выгислениями.

Полученные формулы выглядят сложно, но вычисления можно произвести, используя, например, простейшую программу в пакете символьных вычислений Mathematica. Первые четыре коэффициента (плотности) ядра теплопроводности имеют следующий вид:

$$
\begin{aligned}
\bar{B}_{0}= & 4 \pi r^{2} \\
\bar{B}_{1}= & \frac{8 \pi}{3}\left(r^{2}+r r^{\prime \prime}\right)+8 \pi\left(\xi-\frac{1}{6}\right)\left(-1+r^{2}+2 r r^{\prime \prime}\right) \\
\bar{B}_{2}= & \frac{8 \pi \xi^{2}}{r^{2}}\left(-1+r^{2}+2 r r^{\prime \prime}\right)^{2}+\frac{8 \pi \xi}{3 r^{2}}\left[-1+5 r r^{\prime \prime}+r^{\prime 2}\left(1-7 r r^{\prime \prime}\right)+\right. \\
& \left.+7 r^{2} r^{\prime} r^{(3)}+3 r^{3} r^{(4)}\right]-\frac{2 \pi}{315 r^{2}}\left[34 r^{\prime 4}-354 r r^{\prime 2} r^{\prime \prime}+504 r^{2} r^{\prime} r^{(3)}+\right. \\
& \left.+21\left(-2+r r^{\prime \prime}\left(10+7 r r^{\prime \prime}\right)+10 r^{3} r^{(4)}\right)\right], \\
\bar{B}_{3}= & \frac{16 \pi \xi^{3}}{3 r^{4}}\left(-1+r^{\prime 2}+2 r r^{\prime \prime}\right)^{3}+\frac{8 \pi \xi^{2}}{3 r^{4}}\left[1+9 r^{\prime 6}-r^{\prime 4}\left(17+18 r r^{\prime \prime}\right)-\right.
\end{aligned}
$$




$$
\begin{aligned}
& -6 r^{2} r^{33} r^{(3)}+2 r^{2} r^{\prime}\left(3+14 r r^{\prime \prime}\right) r^{(3)}+r^{2}\left(7-4 r r^{\prime \prime}\left(-7+8 r r^{\prime \prime}\right)+\right. \\
& \left.\left.+6 r^{3} r^{(4)}\right)+2 r\left(-5 r^{\prime \prime}+8 r r^{\prime \prime 2}+5 r^{3} r^{(3)^{2}}+3 r^{2}\left(-1+2 r r^{\prime \prime}\right) r^{(4)}\right)\right]- \\
& -\frac{4 \pi \xi}{315 r^{4}}\left[2 r^{\prime 4}\left(-315+859 r r^{\prime \prime}\right)-1616 r^{2} r^{\prime 3} r^{(3)}+3 r^{\prime 2}\left(196-2 r r^{\prime \prime} \times\right.\right. \\
& \left.\times\left(-35+839 r r^{\prime \prime}\right)+271 r^{3} r^{(4)}\right)+3 r^{2} r^{\prime}\left(2\left(140+677 r r^{\prime \prime}\right) r^{(3)}-\right. \\
& \left.-91 r^{2} r^{(5)}\right)-21\left(-2+24 r r^{\prime \prime}-50 r^{2} r^{\prime \prime 2}+r^{3}\left(-44 r^{\prime \prime 3}+25 r^{(4)}\right)-\right. \\
& \left.\left.-27 r^{4}\left(r^{(3)^{2}}+r^{\prime \prime} r^{(4)}\right)+5 r^{5} r^{(6)}\right)\right]-\frac{\pi}{45045 r^{4}}\left[37364 r^{\prime 6}-\right. \\
& -248156 r r^{\prime 4} r^{\prime \prime}+150332 r^{2} r^{\prime 3} r^{(3)}-13 r^{\prime 2}\left(2772-3 r r^{\prime \prime}(1540+\right. \\
& \left.\left.+10981 r r^{\prime \prime}\right)+4906 r^{3} r^{(4)}\right)+286 r^{2} r^{\prime}\left(\left(-210-1139 r r^{\prime \prime}\right) r^{(3)}+\right. \\
& \left.+111 r^{2} r^{(5)}\right)+143\left(-16+168 r r^{\prime \prime}-420 r^{2} r^{\prime \prime 2}+r^{3}\left(-616 r^{\prime \prime 3}+\right.\right. \\
& \left.\left.\left.+210 r^{(4)}\right)-3 r^{4}\left(47 r^{(3)^{2}}+24 r^{\prime \prime} r^{(4)}\right)+84 r^{5} r^{(6)}\right)\right]
\end{aligned}
$$

В этих формулах функция $r$ зависит от радиальной координаты $\rho$, в то время как коэффициенты ядра теплопроводности с полуцелым индексом

$$
\begin{aligned}
B_{1 / 2}= & -4 \pi^{3 / 2} r^{2}, \\
B_{3 / 2}= & -8 \pi^{3 / 2} \xi\left(-1+r^{2}+2 r r^{\prime \prime}\right)+\frac{\pi^{3 / 2}}{3}\left(-4+3 r^{2}+6 r r^{\prime \prime}\right), \\
B_{5 / 2}= & \frac{-8 \pi^{3 / 2} \xi^{2}}{r^{2}}\left(-1+r^{\prime 2}+2 r r^{\prime \prime}\right)^{2}-\frac{2 \pi^{3 / 2} \xi}{3 r^{2}}\left(-4+4 r^{\prime 2}+20 r r^{\prime \prime}-\right. \\
& \left.-30 r r^{\prime 2} r^{\prime \prime}-12 r^{2} r^{\prime \prime 2}+9 r^{2} r^{\prime} r^{(3)}+6 r^{3} r^{(4)}\right)+\frac{\pi^{3 / 2}}{120 r^{2}}(-32+ \\
& \left.+30 r^{4}+160 r r^{\prime \prime}-315 r r^{\prime 2} r^{\prime \prime}-50 r^{2} r^{\prime \prime 2}+140 r^{2} r^{\prime} r^{(3)}+90 r^{3} r^{(4)}\right)
\end{aligned}
$$

зависят от радиальной функции $r$ на границе, $r=r(R)$. Из уравнений (35) и (36) видно, что коэффишиенты ядра теплопроводности $B_{k}$ и $B_{k+1 / 2}$ являются полиномами по $\xi$ степени $k$.

Хорошо известно [12], что коэффициенты ядра теплопроводности с целым индексом состоят из объемной части и интеграла по поверхности. Мы получили другое представление для этих коэффициентов в виде интеграла по $\rho$. Эти выражения находятся в согласии с известными. Действительно, рассмотрим, например, коэффициент $B_{1}$. В соответствии со стандартной формулой имеем

$$
B_{1}=\left(\frac{1}{6}-\xi\right) \int_{V} \mathcal{R} d V+\left.\frac{1}{3} \int_{S} \operatorname{tr} K d S\right|_{\rho=R}+\left.\frac{1}{3} \int_{S} \operatorname{tr} K d S\right|_{\rho=-R} .
$$

Объемный вклад совпадает с полученным (35б). Поверхностный вклад имеет вид

$$
\left.\frac{1}{3} \int_{S} \operatorname{tr} K d S\right|_{\rho=R}+\left.\frac{1}{3} \int_{S} \operatorname{tr} K d S\right|_{\rho=-R}=\left.\frac{16 \pi}{3} r^{\prime} r\right|_{\rho=R}
$$


Из нашей формулы (35б) получаем такое же выражение

$$
\frac{8 \pi}{3} \int_{-R}^{+R}\left(r^{\prime 2}+r r^{\prime \prime}\right) d \rho=\frac{8 \pi}{3} \int_{-R}^{+R}\left(r r^{\prime}\right)^{\prime} d \rho=\left.\frac{16 \pi}{3} r r^{\prime}\right|_{\rho=R} .
$$

Нетрудно проверить, что коэффициенты ядра теплопроводности вплоть до $B_{2}$ находятся в согласии с обшими выражениями. Для высших индексов в литературе не имеется общих выражений.

В соответствии с [7] достаточное условие сушествования отрицательного минимума энергии нулевых колебаний формулируется в виде условий положительности двух коэффициентов ядра теплопроводности,

$$
\begin{aligned}
& B_{2}=\int_{-\infty}^{+\infty} d \rho \bar{B}_{2}=h_{2,2} \xi^{2}+h_{2,1} \xi+h_{2,0}, \\
& B_{3}=\int_{-\infty}^{+\infty} d \rho \bar{B}_{3}=h_{3,3} \xi^{3}+h_{3,2} \xi^{2}+h_{3,1} \xi+h_{3,0} .
\end{aligned}
$$

Коэффишиенты $h_{k, l}$ зависят от структуры кротовой норы. Таким образом, задача сводится к анализу полиномов по $\xi$ второй и третьей степени, коэффициенты которых зависят от параметров фонового пространства. Кротовые норы различной формы, возможно, могут сушествовать для различных величин константы неминимальной связи $\xi$. Наоборот, для определенной величины $\xi$ полиномы могут быть положительны для специальных форм кротовой норы.

\section{6. МОДЕЛЬ ГОРЛОВИНЫ $r(\rho)=\rho \operatorname{th}(\rho / \tau)+a$}

Мы не будем воспроизводить здесь формулы для коэффишиентов ядра теплопроводности ввиду их громоздкости. Они могут быть получены из обших формул (35). В этой модели есть два параметра: $\tau$ и $\alpha=\tau / a$. Размерный параметр $a$ характеризует в целом этот вид кротовой норы. Малой его величине соответствует малый размер кротовой норы. Безразмерный параметр $\alpha$ характеризует форму кротовой норы - отношение длины горловины к ее радиусу. Заменяя переменную интегрирования $\rho=x a$, получим следуюшие выражения для коэффициентов $B_{2}$ и $B_{3}$ :

$$
\begin{aligned}
& B_{2}=\int_{-\infty}^{+\infty} d \rho \bar{B}_{2}=\frac{1}{a}\left(b_{2,2} \xi^{2}+b_{2,1} \xi+b_{2,0}\right), \\
& B_{3}=\int_{-\infty}^{+\infty} d \rho \bar{B}_{3}=\frac{1}{a^{3}}\left(b_{3,3} \xi^{3}+b_{3,2} \xi^{2}+b_{3,1} \xi+b_{3,0}\right),
\end{aligned}
$$

где $b_{k, l}$ зависят только от $\alpha=\tau / a$. Из (35в) следует, что $b_{2,2}>0$. Таким образом, существует возможность анализа энергии нулевых колебаний для различных величин параметра $\alpha$. Энергия нулевых колебаний для малых размеров кротовой норы $a \rightarrow 0$ ведет себя следуюшим образом:

$$
E^{\mathrm{ren}} \approx-\frac{\ln (m a)^{2}}{32 \pi^{2}} B_{2}=-\frac{\ln (m a)^{2}}{32 \pi^{2} a}\left(b_{2,2} \xi^{2}+b_{2,1} \xi+b_{2,0}\right) .
$$


Используя общее выражение для коэффициента $B_{2}$, можно найти в явном виде полином по $\xi$ в (37) для больших значений $\alpha \rightarrow \infty$ (радиус горловины много меньше ее длины),

$$
E^{\mathrm{ren}} \approx-\frac{\sqrt{\alpha} \ln (m a)^{2}}{240 a}\left(30 \xi^{2}-10 \xi+1\right)
$$

Для малых значений $\alpha \rightarrow 0$ (длина горловины много меньше ее радиуса) имеем

$$
E^{\mathrm{ren}} \approx-\frac{\left(30+\pi^{2}\right) \ln (m a)^{2}}{2700 \pi a \alpha}\left(240 \xi^{2}-80 \xi+7\right)
$$

Зависимость дискриминанта $\Delta=b_{2,1}^{2}-4 b_{2,2} b_{2,0}$ полинома по $\xi$ от $\alpha$ показана на рис. 2 . Из полученной зависимости и уравнений (38), (39) можно заключить, что дискриминант всегда отрицателен для произвольных значений $\alpha$. Это означает, что энергия нулевых колебаний всегда положительна для малых размеров кротовой норы при произвольных значениях константы неминимальной связи $\xi$ и произвольном отношении длины горловины к ее радиусу.

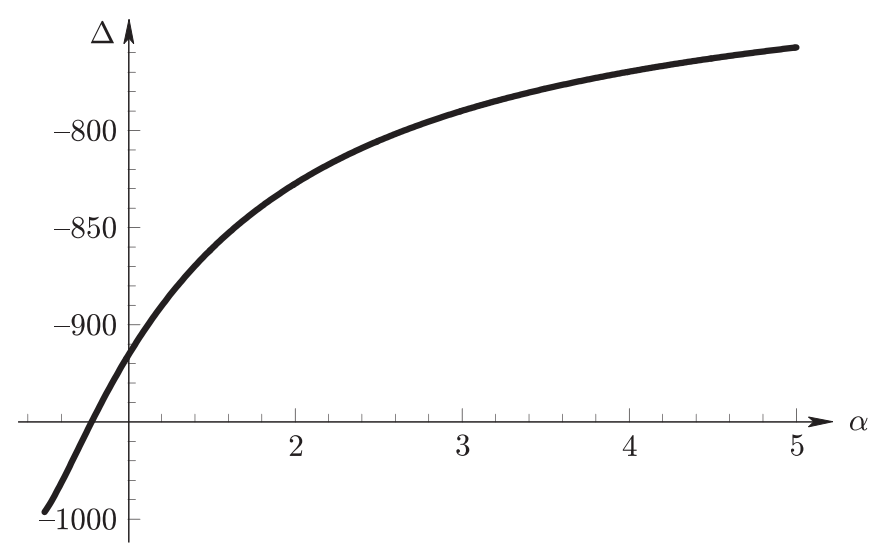

Рис. 2

Поведение энергии нулевых колебаний для больших размеров горловины $(a \rightarrow \infty)$ описывается формулой

$$
E^{\mathrm{ren}} \approx-\frac{B_{3}}{32 \pi^{2} m^{2}}=-\frac{1}{32 \pi^{2} m^{2} a^{3}}\left(b_{3,3} \xi^{3}+b_{3,2} \xi^{2}+b_{3,1} \xi+b_{3,0}\right)
$$

Энергия нулевых колебаний будет иметь минимум при некотором значении $a$, если это выражение будет отрицательным. Рассмотрим полином

$$
P=b_{3,3} \xi^{3}+b_{3,2} \xi^{2}+b_{3,1} \xi+b_{3,0}
$$


для различных значений $\alpha$, начиная с малых $\alpha \ll 1$. Энергия нулевых колебаний будет иметь минимум, если этот полином будет положителен. В пределе $\alpha \rightarrow 0$ приближенно имеем

$$
\begin{aligned}
P \alpha^{3} \approx & \frac{512 \pi\left(45+2 \pi^{2}\right)}{135} \alpha \xi^{3}-\left(\frac{256 \pi\left(21+\pi^{2}\right)}{189}+\frac{256 \pi\left(45+2 \pi^{2}\right)}{135} \alpha\right) \xi^{2}+ \\
& +\left(\frac{512 \pi\left(21+\pi^{2}\right)}{945}+\frac{224 \pi\left(45+2 \pi^{2}\right)}{675} \alpha\right) \xi-\frac{368 \pi\left(21+\pi^{2}\right)}{6615}+\frac{44 \pi\left(45+2 \pi^{2}\right)}{2025} \alpha
\end{aligned}
$$

В этом выражении сохранены члены до $\alpha^{-3}$. Этот полином в пределе $\alpha \rightarrow 0$ имеет два комплексных корня и один действительный

$$
\xi \approx \xi_{0}=\frac{5\left(21+\pi^{2}\right)}{14\left(45+2 \pi^{2}\right)} \frac{1}{\alpha}
$$

Поскольку коэффициент при $\xi^{3}$ положителен, то полином будет положителен для всех $\xi>\xi_{0}$. Для малых значений $\alpha=\tau / a \ll 1$ получаем нижнюю гранишу параметра $\xi$, где кротовые норы, возможно, могут сушествовать. Чем больше $\alpha$, тем меньше нижняя граница $\xi$. Для наглядности на рис. 3 показана зависимость действительных корней полинома $P \alpha^{3}$ от параметра $\alpha=\tau / a$. Вся область значений $\alpha$ естественно разбивается на четыре части: в областях I и IV имеется один действительный и два комплексно-сопряженных корня, а в областях II и III все три корня полинома действительны. В затемненной области полином положителен и кротовые норы могут существовать с соответствуюшими значениями параметров $\alpha$ и $\xi$. Начиная с малых значений $\alpha \ll 1$ и вплоть до значения $\alpha=3.728$ (область I на рис. 3 ) полином имеет один действительный и два комплексно-сопряженных корня. Поэтому сушествует только нижняя гранища $\xi$, которая уменьшается с ростом $\alpha$. В точке $\alpha=3.728$ все три корня полинома становятся действительными и по этой причине появляются две области, где полином положителен. Первая область $0.169<\xi<0.492$, вторая $\xi>0.492$. При дальнейшем увеличении $\alpha$ один из корней полинома неограниченно возрастает, и в точке $\alpha=6.05$ остается только два корня полинома. Дело в том, что в этой точке коэффициент $b_{3,3}=0$, и кубический полином становится квадратичным с положительной областью $-0.024<\xi<0.302$. Для бо́льших значений $\alpha$ получаем верхнюю границу $\xi$, в которой полином положителен, поскольку коэффициент при $\xi^{3}$ отрицателен. Как видно из рис. 3 , начиная с $\alpha=6.05$ появляются две области, где полином положителен. При $\alpha=7.5$ полином имеет один действительный и два комплексно-сопряженных корня, и поэтому остается только одна область, где полином положителен, $\xi<0.289$ (область IV на рис. 3). Для больших значений $\alpha \gg 1$ эта верхняя граница $\xi$ стремится к постоянному значению 0.254 . Действительно, в пределе $\alpha \rightarrow \infty$ получаем

$$
P \approx \pi^{2} \frac{\sqrt{\alpha}}{3}\left(-5 \xi^{3}+\frac{5}{2} \xi^{2}-\frac{1}{2} \xi+\frac{1}{21}\right)
$$




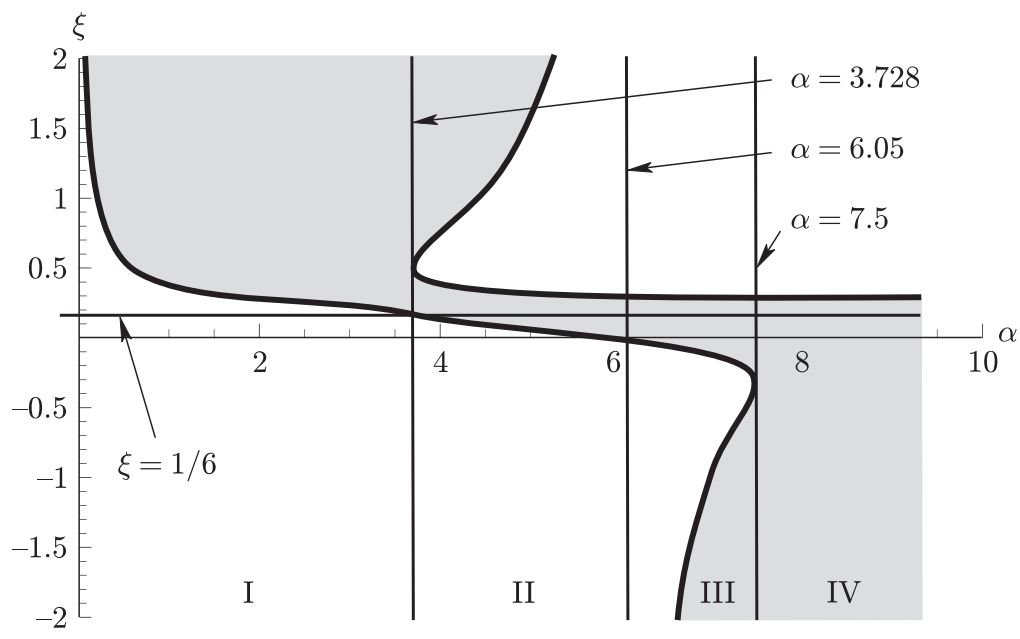

Рис. 3

Этот полином положителен при $\xi<0.254$.

Перейдем теперь к уравнениям Эйнштейна. Энергия, соответствуюшая этой конфигурации, дается выражением

$$
E=-\frac{c^{4}}{2 G} \int_{-\infty}^{\infty} G_{t}^{t} r^{2}(\rho) d \rho=\frac{c^{4}}{4 G} \int_{-\infty}^{\infty} \mathcal{R} r^{2}(\rho) d \rho=-\frac{4 c^{4}}{G}\left(1-\frac{\pi^{2}-6}{36} \alpha\right) a
$$

Приравнивая эту энергию к энергии нулевых колебаний

$$
E^{\mathrm{ren}}=-\frac{\hbar c}{a} f(a m, \alpha, \xi)
$$

получаем уравнение

$$
\left(1-\frac{\pi^{2}-6}{36} \alpha\right) a^{2}=\frac{1}{2} l_{\mathrm{Pl}}^{2} f(a m, \alpha, \xi)
$$

Для того чтобы найти параметры кротовой норы этого вида, необходимо рассмотреть последнее уравнение при минимальном значении энергии, т.е. при максимальном значении функции $f(a m, \alpha, \xi)$. Поскольку эта функция в точке максимума положительна, то кротовая нора может сушествовать при $\alpha<36 /\left(\pi^{2}-6\right)=9.3$. Таким образом, кротовые норы такого вида могут сушествовать при $\tau / a<9.3$. Конкретные значения $\tau, a$ и область $\xi$ можно найти численными расчетами функции $f(a m, \alpha, \xi)$, что выходит за рамки данной работы.

Рассмотрим отдельно специальные значения $\xi$. Скалярное поле с конформной константой неминимальной связи $\xi=1 / 6$ может самосогласованно описывать кротовые норы при $\tau / a \in(3.76,9.3)$ (см. рис.3). Для минимальной связи $\xi=0$ имеем $\tau / a \in(5.74,9.3)$. 


\section{7. ЗАКЛЮЧЕНИЕ}

В данной работе мы обсудили возможность сушествования полуклассических кротовых нор с метрикой (1) и профилем горловины (5). Используемый подход основан на вычислении двух коэффициентов ядра теплопроводности $B_{2}$ и $B_{3}$. Для этого был развит метод вычисления коэффициентов ядра теплопроводности и получено обшее выражение для любого коэффициента ядра теплопроводности оператора Лапласа для пространства (1). Первые семь коэффициентов в явной форме для любого профиля горловины даются выражениями (35) и (36). Достаточным условием возможного сушествования кротовых нор является положительность коэффициентов $B_{2}$ и $B_{3}$. Дополнительные условия следуют из $t-t$ уравнения Эйнштейна.

Энергия нулевых колебаний для малых размеров кротовой норы всегда положительна при произвольных значениях константы неминимальной связи $\xi$. Этот результат противоположен полученному для пространства кротовой норы с бесконечно короткой горловиной [7]. Рассматриваемая модель кротовой норы (1), (5) характеризуется двумя параметрами: $\tau$ - длиной и $a$ - радиусом горловины кротовой норы. В этой модели возможно ввести в рассмотрение длину горловины, поскольку пространство вне горловины норы экпоненциально быстро стремится к плоскому пространству Минковского. Возможное сушествование таких кротовых нор критически зависит от параметра $\xi$ и отношения длины горловины к ее радиусу $\alpha=\tau / a$. Верхняя граниша для параметра $\alpha$ следует из уравнений Эйнштейна: $\alpha<9.3$. Кротовая нора с малой величиной параметра $\alpha$ может быть самосогласованно рассмотрена с учетом квантовых флуктуаций скалярного массивного поля при большой величине $\xi \sim 1 / \alpha$. Скалярное поле с конформной связью $\xi=1 / 6$ может самосогласованно описывать кротовые норы с $\tau / a \in(3.76,9.3)$. Для минимальной связи $\xi=0$ имеем $\tau / a \in(5.74,9.3)$.

Необходимо отметить, что в пределе нулевой длины горловины $\alpha=\tau / a \rightarrow 0$ полученные результаты не согласуются с результатами работы [7]. Дело в том, что в модели, рассмотренной в работе [7], изначально имеется сингулярность. Скалярная кривизна сингулярна на горловине, сушествует сингулярная поверхность коразмерности один. В этом случае в работе [14] были получены обшие формулы для коэффициентов ядра теплопроводности (до $B_{2}$ включительно), которые не могут быть получены из формул для гладкого фона [11]. Причина состоит в том, что коэффициенты ядра теплопроводности определяются как коэффициенты разложения по размерному параметру, который в случае гладкого фона всегда можно сделать меньше параметра, характеризующего фоновое многообразие. Это неверно в случае наличия сингулярных поверхностей, поскольку носителем, например, дельта-функции является множество меры ноль. Данное обстоятельство приводит к другой форме коэффициентов ядра теплопроводности. Более того, в этом пространстве поведение энергии для больших размеров кротовой норы определяется коэффициентом $B_{5 / 2}$.

Возникновение ограничений на константу $\xi$ связано с тем, что эффективная масса $m_{\text {eff }}^{2}=m^{2}+\xi \mathcal{R}$ может менять свой знак. В случае кротовой норы с бесконечно короткой горловиной, рассмотренной в работе [7], скалярная кривизна отрицательна для любых 
значений радиуса горловины, и поэтому существует фиксированная нижняя граница $\xi$. В нашем случае скалярная кривизна

$$
\mathcal{R}=\frac{-6 y^{2}-4 \alpha+\alpha^{2}+\left(4 y^{2}+(-4+\alpha) \alpha\right) \operatorname{ch}(2 y / \alpha)-2 y(-2+3 \alpha) \operatorname{sh}(2 y / \alpha)}{\tau^{2} \operatorname{ch}^{4}(y / \alpha)(1+y \operatorname{th}(y / \alpha))^{2}}
$$

может менять свой знак в зависимости от параметров модели. Для малых $\alpha$ кривизна отрицательна, а начиная с $\alpha=4$ в окрестности $y=0$ появляется область, где кривизна становится положительной. Эта область увеличивается с увеличением $\alpha$, и для достаточно больших $\alpha$ скалярная кривизна становится фактически положительной величиной. Это находится в качественном согласии с изложенным вьше. Действительно, для малых $\alpha$ (отрицательная скалярная кривизна) получаем нижнюю границу $\xi$, и наоборот, для больших значений $\alpha$ (положительная кривизна) получаем верхнюю границу $\xi$.

Благодарности. Работа была частично поддержана Российским фондом фундаментальных исследований, грант № 02-02-17177.

\section{Список литературы}

[1] L. Flamm. Phys. Z. 1916. V. 17. P. 48.

[2] A. Einstein, N. Rosen. Phys. Rev. 1935. V. 48. P. 73.

[3] J. A. Wheeler. Phys. Rev. 1955. V. 97. Р. 511; Дж. А. Уилер. Гравитация, нейтрино и Вселенная. М.: ИЛ, 1962.

[4] M.S. Morris, K.S. Thorne. Am. J. Phys. 1988. V. 56. P. 395; M. S. Morris, K. S. Thorne, U. Yurtsever. Phys. Rev. Lett. 1988. V. 61. P. 1446.

[5] M. Visser. Lorentzian Wormholes: from Einstein to Hawking. Woodbury: American Institute of Physics, 1995.

[6] S. V. Sushkov. Phys. Lett. A. 1992. V. 164. P. 33; D. Hochberg, A. Popov, S. V. Sushkov. Phys. Rev. Lett. 1997. V. 78. P. 2050.

[7] N.R. Khusnutdinov, S. V. Sushkov. Phys. Rev. D. 2002. V. 65. P. 084028.

[8] С. Хокинг, Дж. Эллис. Крупномасштабная структура пространства-времени. М.: Мир, 1977.

[9] S. V. Sushkov. Gravit. Cosmol. 2001. V. 7. P. 194.

[10] J.S. Dowker, R. Critchley. Phys. Rev. D. 1976. V. 13. P. 3224; S. W. Hawking. Commun. Math. Phys. 1977. V. 55. P. 133; S. K. Blau, M. Visser, A. Wipf. Nucl. Phys. B. 1988. V. 310. P. 163.

[11] E. Elizalde, S. D. Odintsov, A. Romeo, A.A. Bytsenko, S. Zerbini. Zeta Regularization Techniques with Applications. Singapore: World Scientific, 1994.

[12] M. Bordag. J. Phys. A. 1995. V. 28. P. 755; M. Bordag, K. Kirsten. Phys. Rev. D. 1996. V. 53. P. 5753; M. Bordag, K. Kirsten, E. Elizalde. J. Math. Phys. 1996. V. 37. P. 895; M. Bordag, E. Elizalde, K. Kirsten, S. Leseduarte. Phys. Rev. D. 1997. V. 56. P. 4896; M. Bordag, K. Kirsten, D. Vassilevich. Phys. Rev. D. 1999. V. 59. P. 085011.

[13] N. R. Khusnutdinov, M. Bordag. Phys. Rev. D. 1999. V. 59. P. 064017.

[14] P. B. Gilkey, K. Kirsten, D. V. Vassilevich. Nucl. Phys. B. 2001. V. 601. P. 125.

[15] D. V. Fursaev. Phys. Lett. B. 1994. V. 334. P. 53.

[16] E. R. Bezerra de Mello, V.B. Bezerra, N.R. Khusnutdinov. J. Math. Phys. 2001. V. 42. P. 562 . 